\title{
COUPLED FINITE AND BOUNDARY ELEMENT METHOD FOR SOLVING MAGNETIC HYSTERESIS PROBLEMS
}

\author{
ILYA STUPAKOV, MIKHAIL ROYAK \& NATALIA KONDRATYEVA \\ Novosibirsk State Technical University, Russia
}

\begin{abstract}
This paper is devoted to effective solving of magnetic hysteresis problems in 3D modeling of accelerator magnets. It is proposed to use boundary elements and a reduced scalar magnetic potential in the air and finite elements, in addition to a full scalar magnetic potential in ferromagnetic materials. There is no need to mesh the air domain by using that approach. Also, it allows nonlinear and inhomogeneous magnetization in ferromagnets to be taken into account. Numerical simulation is carried out using three different models of hysteresis: the Preisach model, the model based on dry friction and the Vinokurov model. To verify the accuracy of the developed approach, the simulation results are compared with the magnetic field measurements for the deflecting accelerator magnet.
\end{abstract}

Keywords: finite elements, boundary elements, magnetic field, hysteresis, total and reduced potential.

\section{INTRODUCTION}

Designing acceleration magnets requires effective solving of magnetostatic problems. The most often used method to solve such problems is the finite element method. Employing the boundary element method to solve magnetostatic problems is proposed in two studies by Andjelic et al. [1], [2]. However, even though the boundary element method significantly simplifies the mesh construction, it can only account for nonlinear properties of ferromagnetic materials in ineffective way, while taking nonlinearity into account is important for obtaining an accurate solution of real problems. Thus, the coupled approach with the use of both finite and boundary elements is gaining popularity in recent years [3], [4]. Most notably, this approach is used in CERN and is described in Russenschuck [5]. However, the approach presented in this book employs vector magnet potential. This is less effective for solving various problems than the approach based on two scalar potentials - total and reduced. This idea was first proposed in Simkin and Trowbridge [6], but the algorithm used for combining these potentials was overcomplicated which led to low popularity of the method. In this paper, we propose a simplified formulation with two potentials for the coupled finite and boundary element method.

One more difference from the existing works is the employment of magnetization instead of magnetic permeability. This enables us to account for hysteresis effects in ferromagnetic materials, which significantly improves the accuracy of modeling in some situations. This paper presents a comparison of computational results using several hysteresis models with the magnetic field measurements obtained from a physics experiment.

\section{MATHEMATICAL MODEL}

Let us consider quasistatic magnetic problems, i.e. the problems where eddy currents and displacement currents are not taken into account. Those problems are described by a special case of Maxwell's equations:

$$
\begin{aligned}
& \nabla \times \mathbf{H}=\mathbf{J}, \\
& \nabla \cdot \mathbf{B}=\mathbf{0},
\end{aligned}
$$

where $\mathbf{H}$ is magnetic field intensity, $\mathbf{B}$ is magnetic induction, and $\mathbf{J}$ is external current density. 
Instead of a traditional relation between $\mathbf{H}$ and $\mathbf{B}$, i.e. $\mathbf{B}=\mu \mathbf{H}$, let us use a more general one:

$$
\mathbf{B}=\mu_{0}(\mathbf{H}+\mathbf{M}),
$$

where $\mu_{0}$ is magnetic permeability of vacuum, and $\mathbf{M}$ is magnetization. Let us consider that the computational domain $\Omega$ is simply connected, and its boundary $\partial \Omega$ can be divided into two parts $\Gamma_{H}$ and $\Gamma_{B}$, on which homogeneous boundary conditions are specified:

$$
\begin{gathered}
\mathbf{H} \times\left.\mathbf{n}\right|_{\Gamma_{H}}=0, \\
\left.\mathbf{B} \cdot \mathbf{n}\right|_{\Gamma_{B}}=0,
\end{gathered}
$$

where $\mathbf{n}$ is an external normal to the domain. Let us seek $\mathbf{H}$ in the form of

$$
\mathbf{H}=\mathbf{H}_{\text {ext }}-\nabla u,
$$

where $u$ is a scalar magnetic potential, and $\mathbf{H}_{\text {ext }}$ is the external field that should satisfy the condition of eqn (7):

$$
\nabla \times \mathbf{H}_{\text {ext }}=\mathbf{J} .
$$

A solution of the magnetostatic equations in an unbounded homogeneous domain can be used as the external field. It can be obtained in an explicit form of the Biot-Savart Law [7]:

$$
\mathbf{H}_{\text {ext }}(\mathbf{x})=\frac{1}{4 \pi} \int_{\Omega} \frac{\mathbf{J} \times(\mathbf{x}-\mathbf{y})}{|\mathbf{x}-\mathbf{y}|^{3}} d \mathbf{y} .
$$

Note that the field $\mathbf{H}_{\text {ext }}$ presented in the way above also satisfies eqn (9):

$$
\nabla \cdot \mathbf{H}_{\text {ext }}=0 .
$$

Since the domain $\Omega$ is simply connected, the existence of the scalar potential $u$ satisfying eqn (9) follows from $\nabla \times\left(\mathbf{H}-\mathbf{H}_{\text {ext }}\right)=0$ according to Helmholtz's theorem. Thus, eqn (1) is fulfilled and one needs to seek for potential satisfying eqn (2). The obtained scalar potential is called a reduced potential because to calculate the magnetic field one needs to add the external field $\mathbf{H}_{\text {ext }}$ to it. In case $\mathbf{M}$ is dependent on field $\mathbf{H}$, this could be inconvenient because of significant computational costs required to obtain field $\mathbf{H}_{\text {ext }}$. To avoid this, the formulation with coupled total and reduced scalar potentials can be used. Let us show that an analogous formulation can be obtained in a simpler way than that described in Simkin and Trowbridge [6]. To do that, it is enough to modify the external field $\mathbf{H}_{\text {ext }}$. Let us divide the computational domain $\Omega$ into two parts: domain $\Omega_{0}$ with no magnetic materials $(\mathbf{M}=\mathbf{0})$, and domain $\Omega_{M}$ with no currents $(\mathbf{J}=\mathbf{0})$. Let $\Gamma_{I}$ be the interface between these domains. Fig. 1 shows the described division.

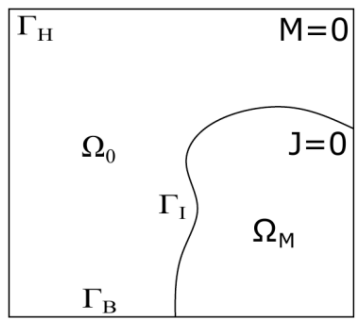

Figure 1: The division of the computational domain. 
Let us require that the modified external field $\widetilde{\mathbf{H}}_{\text {ext }}$ not only satisfies eqn (7) but also vanishes in the domain $\Omega_{\mathrm{M}}$. Thus, field $\mathbf{H}$ in this domain is defined by the scalar potential only, i.e. the potential becomes total in this domain.

To modify the external field in this way, it is sufficient to ensure that eqn (7) is fulfilled in the domain $\Omega_{0}$ and the tangential part of the external field vanishes on the interface $\Gamma_{\mathrm{I}}$. This can be done if the external field is represented in the following way:

$$
\widetilde{\mathbf{H}}_{\text {ext }}=\mathbf{H}_{\text {ext }}-\nabla h,
$$

where function $h$ should satisfy the condition

$$
\nabla h \times\left.\mathbf{n}\right|_{\Gamma_{I}}=\mathbf{H}_{e x t} \times\left.\mathbf{n}\right|_{\Gamma_{I}} .
$$

Lemma 1: A continuous function $h$ satisfying eqn (11) exists if, and only if, the integral of the currents bounded by a closed contour $C$ is equal to zero for every $C$ lying in $\Gamma_{\mathrm{I}}$.

Proof: From the theorem about magnetic field circulation (integral representation of eqn (7)), it follows that a circulation of $\mathbf{H}_{\text {ext }}$ around any contour is equal to the integral of the currents bounded by that contour. Considering this, the lemma is equivalent to the following: for the existence of the function $h$ it is necessary and sufficient that the circulation of $\mathbf{H}_{\text {ext }}$ around any contour lying in $\Gamma_{\mathrm{I}}$ was equal to zero. Necessity follows from the circulation of the gradient of a continuous function being equal to zero.

Sufficiency can be proved constructively. Firstly, let us consider a case when the boundary $\Gamma_{\mathrm{I}}$ is connected. Let us select some point on the boundary where $h$ will be assumed to vanish. From the circulation of $\mathbf{H}_{\text {ext }}$ being equal to zero it follows that the work of the vector field $\mathbf{H}_{\text {ext }}$ is independent from the integration path. Thus, the value of the function $h$ in an arbitrary point can be defined as the work of $\mathbf{H}_{\text {ext }}$ from this point to the point where $h$ is equal to zero. In case the boundary is not connected, $h$ could be analogously defined on each connected component. Outside of the surface $\Gamma_{\mathrm{I}}$, the function $h$ can be extended into the domain $\Omega_{0}$ in any continuous way.

Also note that the sufficient condition for the existence of $h$ is $\Omega_{\mathrm{M}}$ being simply connected. However, this condition is not necessary, e.g., Fig. 2 shows a domain $\Omega_{\mathrm{M}}$ for which conditions of Lemma 1 are fulfilled, thus, function $h$ exists. Note that the conditions of existence of function $h$ almost do not reduce the applicability of the proposed method. This is due to the fact, that if the contour around the current exists inside the magnetic material, it is usually the case when the effects of eddy currents are significant and should be taken into account.

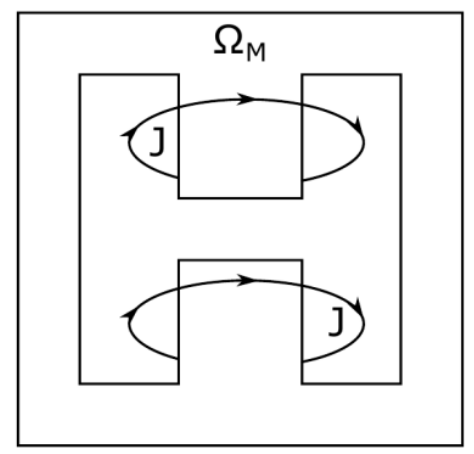

Figure 2: Example of not simply connected domain $\Omega_{\mathrm{M}}$. 
In practice, instead of calculating the work, function $h$ can be obtained as a solution of a best approximation problem

$$
h=\arg \min _{t}\left\|\mathbf{H}_{e x t}-\nabla t\right\|_{L_{2}\left(\Gamma_{I}\right)} .
$$

\section{VARIATIONAL FORMULATION}

To obtain a solution of the magnetostatic problem, one need to seek potential $u$ that satisfies eqn (2) in the domains $\Omega_{0}$ and $\Omega_{M}$. In addition, the continuity of the normal part of $\mathbf{B}$ should be ensured on the interface between the domains. Firstly, let us consider the domain $\Omega_{M}$. In this domain, the potential is total, and eqn (2) takes the form

$$
\nabla \cdot(-\nabla u+\mathbf{M})=0 .
$$
form

The normal part of the field $\mathbf{B}$ on the boundary of the domain $\Omega_{M}$ can be presented in the

$$
\frac{1}{\mu_{0}} \mathbf{B} \cdot \mathbf{n}=-\frac{\partial u}{\partial n}+\mathbf{M} \cdot \mathbf{n} .
$$

In this domain, we use the finite element method for the approximation of the potential. To do so, we rewrite eqn (13) in the weak form using the Galerkin method

$$
(\nabla \cdot(-\nabla u+\mathbf{M}), v)_{\Omega_{M}}=0,
$$

where $(\cdot, \cdot)_{X}$ denotes an inner product of the space $L_{2}(X), u, v \in H_{0}^{1}\left(\Omega_{M}\right)$, and $H_{0}^{1}$ is a Sobolev space which is defined as follows:

$$
H_{0}^{1}\left(\Omega_{M}\right)=\left\{f\left|f \in L_{2}\left(\Omega_{M}\right), \nabla f \in L_{2}\left(\Omega_{M}\right), f\right|_{\Gamma_{H}}=0\right\} .
$$

We integrate eqn (15) by parts

$$
\left(-\frac{\partial u}{\partial n}+\mathbf{M} \cdot \mathbf{n}, v\right)_{\partial \Omega_{M}}=(-\nabla u+\mathbf{M}, \nabla v)_{\Omega_{M}} .
$$

We replace the left part with the normal part of $\mathbf{B}$, according to eqn (14), and employ the boundary conditions. After that, we have

$$
\left(\frac{1}{\mu_{0}} \mathbf{B} \cdot \mathbf{n}, v\right)_{\Gamma_{I}}=(\mathbf{M}, \nabla v)_{\Omega_{M}}-(\nabla u, \nabla v)_{\Omega_{M}}
$$

Secondly, let us consider the domain $\Omega_{0}$. In this domain, the potential is reduced. If we extend function $h$ in a way that it is harmonic, then eqn (2) in this domain is reduced to the Laplace's equation

$$
\Delta u=0 .
$$

The normal part of the field $\mathbf{B}$ on the boundary of the domain $\Omega_{0}$ can be presented in the form

$$
\frac{1}{\mu_{0}} \mathbf{B} \cdot \mathbf{n}=\mathbf{H}_{e x t} \cdot \mathbf{n}-\frac{\partial h}{\partial n}-\frac{\partial u}{\partial n} .
$$

We use the boundary element method for the approximation of the potential in this domain. The approach used is based on Steinbach [8]. We will employ the fact that the solution of the Laplace's equation can be presented in the form

$$
u(y)=\int_{\partial \Omega_{0}} U^{*}(\mathbf{x}, \mathbf{y}) \frac{\partial}{\partial n} u(\mathbf{x}) d S-\int_{\partial \Omega_{0}} \frac{\partial}{\partial n_{x}} U^{*}(\mathbf{x}, \mathbf{y}) u(x) d S_{x},
$$


where $U^{*}(\mathbf{x}, \mathbf{y})$ is the fundamental solution of the Laplace's equation. From this equation, we can obtain the boundary integral equations

$$
\begin{aligned}
u & =\mathrm{V} \frac{\partial u}{\partial n}+\left(\frac{1}{2} \mathrm{I}-\mathrm{K}\right) u, \\
\frac{\partial u}{\partial n} & =\left(\frac{1}{2} \mathrm{I}+\mathrm{K}^{\prime}\right) \frac{\partial u}{\partial n}+\mathrm{D} u,
\end{aligned}
$$

where $\mathrm{V}$ is a single layer potential operator, $\mathrm{K}$ is a double layer potential operator, and $\mathrm{D}$ is a hypersingular integral operator.

After substituting the flows in the second equation by the expression from the first equation, we can obtain the equation for a relation between $u$ and $\frac{\partial u}{\partial n}$ :

$$
\frac{\partial u}{\partial n}=\left(\mathrm{D}+\left(\frac{1}{2} \mathrm{I}+\mathrm{K}^{\prime}\right) \mathrm{V}^{-1}\left(\frac{1}{2} \mathrm{I}+\mathrm{K}\right)\right) u=\mathrm{S} u,
$$

where $\mathrm{S}$ is the Steklov-Poincare operator.

We rewrite eqn (20) in the weak form using the Galerkin method

$$
\left(\frac{1}{\mu_{0}} \mathbf{B} \cdot \mathbf{n}, v\right)_{\partial \Omega_{0}}=\left(\mathbf{H}_{e x t} \cdot \mathbf{n}, v\right)_{\partial \Omega_{0}}-\left(\frac{\partial h}{\partial n}, v\right)_{\partial \Omega_{0}}-\left(\frac{\partial u}{\partial n}, v\right)_{\partial \Omega_{0}},
$$

where $u, v \in H_{0}^{1 / 2}\left(\partial \Omega_{0}\right)$, and $H_{0}^{1 / 2}$ is a Sobolev space which is defined as follows:

$$
H_{0}^{1 / 2}\left(\partial \Omega_{0}\right)=\left\{f\left|\exists g \in H^{1}\left(\Omega_{0}\right): g\right|_{\partial \Omega_{0}}=f,\left.f\right|_{\Gamma_{H}}=0\right\} .
$$

Using the Steklov-Poincare operator, this equation can be transformed into the following form of eqn (27):

$$
\left(\frac{1}{\mu_{0}} \mathbf{B} \cdot \mathbf{n}, v\right)_{\Gamma_{I}}=\left(\mathbf{H}_{e x t} \cdot \mathbf{n}, v\right)_{\Gamma_{I}}-(\mathrm{S} h, v)_{\Gamma_{I}}-(\mathrm{S} u, v)_{\partial \Omega_{0}}
$$

To obtain the final equation, one needs to equate the normal components of $\mathbf{B}$ :

$$
(\mathrm{S} u, v)_{\partial \Omega_{0}}+(\nabla u, \nabla v)_{\Omega_{M}}=\left(\mathbf{H}_{e x t} \cdot \mathbf{n}, v\right)_{\Gamma_{I}}-(\mathrm{S} h, v)_{\Gamma_{I}}+(\mathbf{M}, \nabla v)_{\Omega_{M}},
$$

where $u, v \in H_{0}^{1}\left(\Omega_{M}\right) \cup H_{0}^{1 / 2}\left(\partial \Omega_{0}\right)$.

\section{DISCRETIZATION INTO SYSTEM OF EQUATIONS}

To seek $u$ using the coupled finite and boundary element method, let us introduce a finite dimensional subspace $V_{h}$ of the space $H_{0}^{1}\left(\Omega_{M}\right) \cup H_{0}^{1 / 2}\left(\partial \Omega_{0}\right)$. Let the functions $\psi_{i} \in V_{h}$ form a basis of the space $V_{h}$. Then the projections of the potential $u$ and the function $h$ onto the space $V_{h}$ can be presented in the following form:

$$
u=\sum_{j} q_{j} \psi_{j}, \quad h=\sum_{j} r_{j} \psi_{j},
$$

where $q_{j}$ and $r_{j}$ denote weights of basis functions. Then we can rewrite eqn (28) as a system of equations:

$$
\left(\mathbf{S}^{B E M}+\mathbf{S}^{F E M}\right) \mathbf{q}=\mathbf{f}^{H}-\mathbf{S}^{B E M} \mathbf{r}+\mathbf{f}^{M},
$$

where matrix $\mathbf{S}^{B E M}$ is an approximation of Steklov-Poincare boundary element operator, $\mathbf{S}^{F E M}$ is a finite element stiffness matrix,

$$
f_{i}^{H}=\left(\mathbf{H}_{\text {ext }} \cdot \mathbf{n}, \psi_{i}\right)_{\Gamma_{I}},
$$




$$
f_{i}^{M}=\left(\mathbf{M}, \nabla \psi_{i}\right)_{\Omega_{M}} .
$$

Let us consider the calculation of the contributions from the magnetization $f_{i}{ }^{M}$. Let the domain $\Omega_{M}$ be divided into finite elements $\Omega_{k}$. We assume the magnetization is constant on elements $\Omega_{k}$ and also is dependent on the mean value of the field $\mathbf{H}$ on this element

$$
\widehat{\mathbf{H}}_{k}=\frac{1}{\left|\Omega_{k}\right|} \int_{\Omega_{k}} \mathbf{H} d \Omega,
$$

where $\left|\Omega_{k}\right|$ is the volume of the element $\Omega_{k}$. If we denote

$$
\mathbf{G}_{j}^{k}=\int_{\Omega_{k}} \nabla \psi_{j} d \Omega,
$$

then the mean value of $\mathbf{H}$ can be calculated using eqn (35):

$$
\widehat{\mathbf{H}}_{k}=-\frac{1}{\left|\Omega_{k}\right|} \sum_{j} \mathbf{G}_{j}^{k} q_{j}
$$

Then the contributions from the magnetization $f_{i}^{M}$ can be written as

$$
f_{i}^{M}=\sum_{k} \mathbf{M}\left(\widehat{\mathbf{H}}_{k}\right) \cdot \mathbf{G}_{i}^{k} .
$$

Eqn (30) is nonlinear, because contributions $f_{i}^{M}$ are dependent on the vector of weights q. Let us define a residual vector, whose norm should be minimized

$$
\mathbf{R}(\mathbf{q})=\left(\mathbf{S}^{B E M}+\mathbf{S}^{F E M}\right) \mathbf{q}-\mathbf{f}^{H}+\mathbf{S}^{B E M} \mathbf{r}-\mathbf{f}^{M}(\mathbf{q}) .
$$

To minimize the residual, we use the Gauss-Newton method

$$
\begin{gathered}
\mathbf{J} \mathbf{p}_{m}=-\mathbf{R}\left(\mathbf{q}_{m-1}\right), \\
\lambda_{m}=\arg \min _{\lambda}\left\|\mathbf{R}\left(\mathbf{q}_{m-1}+\lambda \mathbf{p}_{m}\right)\right\|, \\
\mathbf{q}_{m}=\mathbf{q}_{m-1}+\lambda_{m} \mathbf{p}_{m},
\end{gathered}
$$

where $m$ is a sequence number of the iteration over nonlinearity, and a vector of zeros can be used for $\mathbf{q}_{0}$. Matrix $\mathbf{J}$ is calculated using the weights from the previous iteration via eqn (41):

$$
\mathbf{J}=\left(\mathbf{S}^{B E M}+\mathbf{S}^{F E M}\right)-\frac{\partial \mathbf{f}^{M}}{\partial \mathbf{q}}
$$

where the following eqn (42) can be used to calculate the derivatives of $f_{i}^{M}$

$$
\frac{\partial f_{i}^{M}}{\partial q_{j}}=-\frac{1}{\left|\Omega_{k}\right|} \sum_{k}\left(\mathbf{G}_{i}^{k}\right)^{T} \frac{\partial \mathbf{M}}{\partial \mathbf{H}} \mathbf{G}_{j}^{k} .
$$

Note that if the tensor $\frac{\partial \mathbf{M}}{\partial \mathbf{H}}$ is symmetric, the matrix $\mathbf{J}$ will also be symmetric.

\section{HYSTERESIS MODELS}

To solve the classic problem of magnetostatics, one can employ $\mathbf{M}(\mathbf{H})=(\mu-1) \mathbf{H}$. However, if hysteresis effects should be accounted for, one needs to use a hysteresis model, which describes the relation $\mathbf{M}(\mathbf{H})$. There are a lot of different models and none of them are unquestionably the best [9], [10]. In this paper, we consider the three following models. 


\subsection{Preisach model}

The Preisach model is one of the most well-known models of hysteresis [12]. Let us use the Preisach model in the form with a one-dimensional distribution for which the Everett function can be analytically calculated [13], [14]. A one-dimensional distribution is given by the following expression:

$$
\varphi(x)=\frac{a e^{-b x}}{\left(1+c e^{-b x}\right)^{2}}
$$

where $a, b$ and $c$ are model parameters. The magnetization in this model can be expressed as

$$
M(H)=\iint \varphi\left(-h_{1}\right) \varphi\left(h_{2}\right) \gamma_{H}\left(h_{1}, h_{2}\right) d h_{1} d h_{2},
$$

where $\gamma_{H}\left(h_{1}, h_{2}\right)$ can be +1 or -1 depending on the history of $H$. This expression can be represented as a combination of the Everett function values in some points. The Everett function can be obtained analytically by calculating the following integrals:

$$
E(x, y)=\int_{x}^{y} \int_{x}^{h_{2}} \varphi\left(-h_{1}\right) \varphi\left(h_{2}\right) d h_{1} d h_{2} .
$$

To obtain a vector model, let us use the method proposed in Mayergoyz [15]:

$$
\mathbf{M}(\mathbf{H})=\sum_{k} w_{k} \mathbf{r}_{k} M\left(\mathbf{H} \cdot \mathbf{r}_{k}\right),
$$

where $w_{k}$ and $\mathbf{r}_{k}$ represent the weights and points of some unit sphere quadrature, respectively.

\subsection{Vinokurov model}

The Vinokurov model was proposed in Vinokurov et al. [16]. Let us use this model in the form of eqn (47):

$$
M(H)=\sum_{k} w_{k} M_{s}\left(1-\frac{1}{\sqrt{1+2 \frac{\chi_{0}}{M_{S}}\left|H_{e, k}\right|}}\right) \frac{H_{e, k}}{\left|H_{e, k}\right|},
$$

where $w_{k}, M_{s}$ and $\chi_{0}$ are model parameters and $H_{e, k}$ can be obtained using the following update rule:

$$
H_{e, k}^{\text {new }}=\left\{\begin{array}{cc}
H \cos \left(\theta_{k}\right)-H_{c, k} & \left|H \cos \left(\theta_{k}\right)-H_{c, k}\right|>H_{e, k} \\
H \cos \left(\theta_{k}\right)+H_{c, k} & \left|H \cos \left(\theta_{k}\right)+H_{c, k}\right|<H_{e, k}, \\
H_{e, k} & \text { otherwise }
\end{array}\right.
$$

where $\theta_{k}$ and $H_{c, k}$ are model parameters. The vector variant of Vinokurov model will be used in eqn (46).

\subsection{Dry friction model}

The main idea of the dry friction model was proposed by Bergqvist [17]. The difference of this model from two previous models is that the Bergqvist's model is a vector model by definition.

Let us use the modification described in Henrotte et al. [18] and Henrotte and Hameyer [19]. In this model, the reversible component of $\mathbf{H}$ is expressed as

$$
\mathbf{H}_{r}=\sum_{k} w_{k} \mathbf{H}_{r, k},
$$


and $\mathbf{H}_{r, k}$ can be obtained by the following update rule:

$$
\mathbf{H}_{r, k}^{\text {new }}=\left\{\begin{array}{cc}
\mathbf{H}_{r, k} & \left|\mathbf{H}-\mathbf{H}_{r, k}\right| \leq r_{k}, \\
\mathbf{H}-r_{k} \frac{\mathbf{H}-\mathbf{H}_{r, k}}{\left|\mathbf{H}-\mathbf{H}_{r, k}\right|} & \text { otherwise, }
\end{array}\right.
$$

where $w_{k}$ and $r_{k}$ are model parameters. The magnetization can be calculated by the following expression:

$$
\mathbf{M}(\mathbf{H})=M_{S} L\left(\frac{\left|\mathbf{H}_{r}\right|}{a}\right) \frac{\mathbf{H}_{r}}{\left|\mathbf{H}_{r}\right|},
$$

where

$$
L(x)=\operatorname{coth}(x)-\frac{1}{x}
$$

is the Langevin function, $M_{S}$ and $a$ is a model parameter.

\section{NUMERICAL EXPERIMENTS}

To compare these three models, let us use the experimental measurements for a deflecting accelerator magnet described in Royak et al. [11]. The 3D model of this magnet with current coils is shown in Fig. 3.

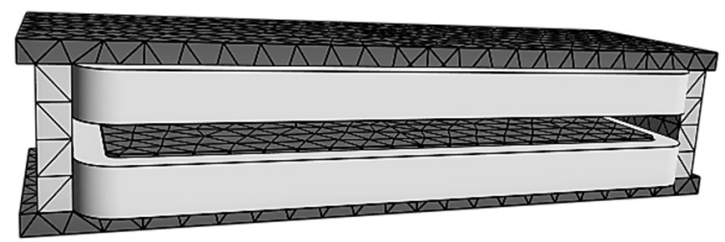

Figure 3: The 3D model of the deflecting accelerator magnet with current coils.

This magnet consists of two materials. For the first material, constructional steel 3, there are some measurements described in Vinokurov et al. [16] that allow us to obtain hysteresis model parameters of the material. The second material is used for the magnet pole. The material is low carbon steel that is close to the pure iron in the chemical composition and magnetic properties. However, numerical simulation was complicated due to exhaustion of samples of this steel; for this reason, we had to determine the hysteresis model parameters by three-dimensional modeling. To select model parameters, a symmetric variation in the current was chosen. The current variation is presented in Fig. 4.
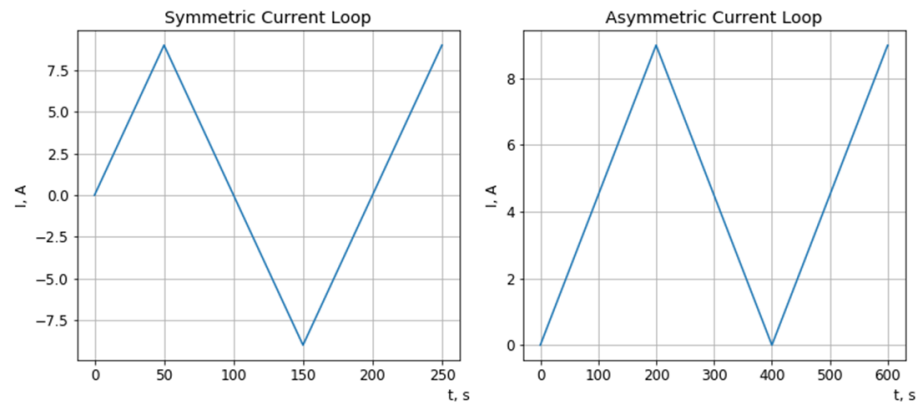

Figure 4: Current loops. 
Then we will compare models both on the symmetric variation in the current and on an asymmetric variation in the current. The hysteresis loops of all three models and measurements for the symmetric current are presented in Fig. 5.

The parameters of pole material for all three models are found by three-dimensional modeling for this loop. As seen, all three models have slight differences from the experimental measurements.
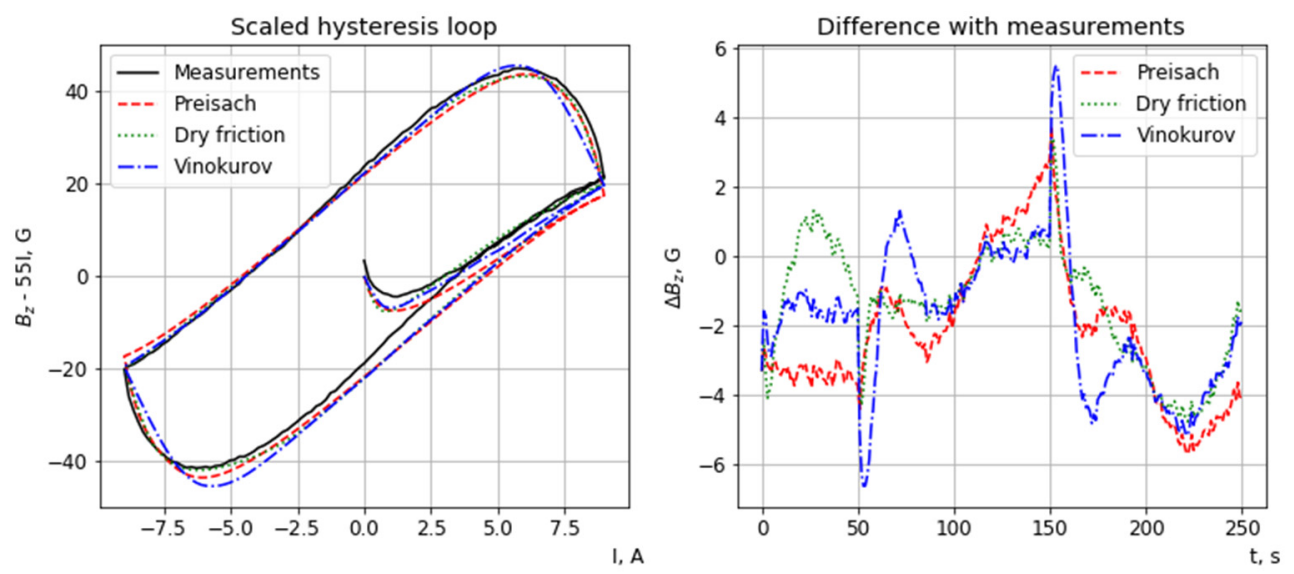

Figure 5: Result on symmetric loop.

The hysteresis loops of all three models and measurements for the asymmetric current are presented in Fig. 6. The parameters of pole material for all three models are the same as for the previous modeling for the symmetric loop. As seen, all three models also have slight differences from the experimental measurements.
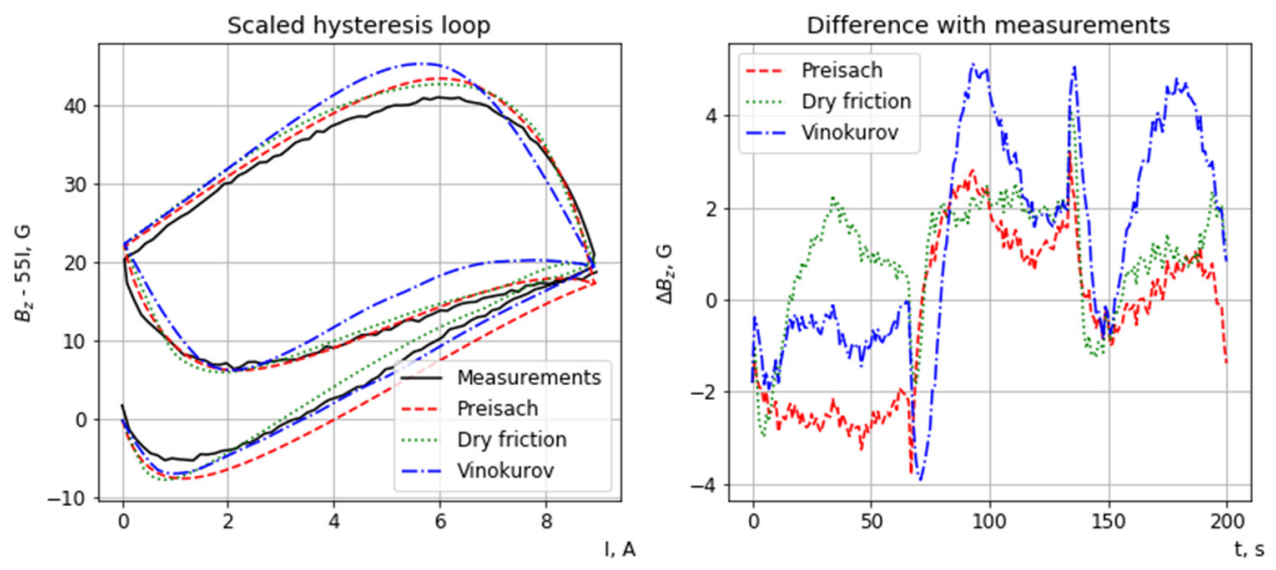

Figure 6: Result on asymmetric loop.

Finally, let us present all results to compare in Table 1. The "time" column in this table represents the time of the whole loop simulation for a model. 
Table 1: Models comparison.

\begin{tabular}{|l|c|c|c|c|}
\hline \multirow{2}{*}{ Model } & \multicolumn{2}{|c|}{ Symmetric loop } & \multicolumn{2}{c|}{ Asymmetric loop } \\
\cline { 2 - 5 } & error, $G$ & time, $s$ & error, $G$ & time, $s$ \\
\hline Preisach & 4.26 & 141 & 3.08 & 84 \\
\hline Vinokurov & 4.17 & 154 & 4.00 & 129 \\
\hline Dry friction & 3.16 & 115 & 2.70 & 103 \\
\hline
\end{tabular}

\section{CONCLUSION}

The results of the numerical experiments show that the proposed approach based on the coupled finite and boundary element method is viable and can be used to solve real physics problems. The promising outlook of the approach is also supported by the fact that even on the coarse mesh used in the experiments, the difference between computational results and the magnetic field measurements is around $1 \%$, which is comparable to the error of the measurements.

All three hysteresis models have slight differences from the measurements for both current loops, so we cannot choose the best model by the accuracy criteria. The simulation times for all models are not significantly different, so time cannot be chosen as a criterion either. But the number of history parameters that corresponds to the computer memory consumption differs significantly. For the results presented in this paper, the minimal memory ( 2 vectors per finite element) was used by the dry friction model and other models used much more memory ( 2 float numbers for each sphere quadrature node per element for the Vinokurov model and not less than 3 floats for each sphere quadrature node per element for the Preisach model).

\section{ACKNOWLEDGEMENT}

The research was fully supported by the Russian Science Foundation grant (project 17-7110203).

\section{REFERENCES}

[1] Andjelic, Z., Of, G., Steinbach, O. \& Urthaler, P., Boundary element methods for magnetostatic field problems: A critical view. Computing and Visualization in Science, 14(3), pp. 117-130, 2011.

[2] Andjelic, Z., Of, G., Steinbach, O. \& Urthaler, P., Fast boundary element methods for industrial applications in magnetostatics. Fast Boundary Element Methods in Engineering and Industrial Applications, Springer: Berlin and Heidelberg, pp. 111143, 2012.

[3] Pusch, D. \& Ostrowski, J., Robust FEM/BEM coupling for magnetostatics on multiconnected domains. IEEE Transactions on Magnetics, 46(8), pp. 3177-3180, 2010.

[4] Bruckner, F. et al., 3D FEM-BEM-coupling method to solve magnetostatic Maxwell equations. Journal of Magnetism and Magnetic Materials, 324(10), pp. 1862-1866, 2012.

[5] Russenschuck, S., Field Computation for Accelerator Magnets: Analytical and Numerical Methods for Electromagnetic Design and Optimization, John Wiley \& Sons, 2011. 
[6] Simkin, J. \& Trowbridge, C.W., Three-dimensional nonlinear electromagnetic field computations, using scalar potentials. IEE Proceedings B-Electric Power Applications, IET, vol. 127, no. 6, pp. 368-374, 1980.

[7] Stupakov, I.M., Royak, M.E. \& Kondratyeva, N.S., The method for calculating magnetic field induced by current coils. 13th International Scientific-Technical Conference on Actual Problems of Electronics Instrument Engineering (APEIE), vol. 2, pp. 347-350, 2016.

[8] Steinbach, O., Numerical approximation methods for elliptic boundary value problems: finite and boundary elements. Springer Science \& Business Media, 2007.

[9] Liorzou, F., Phelps, B. \& Atherton, D.L., Macroscopic models of magnetization. IEEE Transactions on Magnetics, 36(2), pp. 418-428, 2000.

[10] Benabou, A., Clénet, S. \& Piriou, F., Comparison of Preisach and Jiles-Atherton models to take into account hysteresis phenomenon for finite element analysis. Journal of Magnetism and Magnetic Materials, 261(1-2), pp. 139-160, 2003.

[11] Royak, M.E. et al., Application of a new model of residual magnetization of iron for calculating the deflecting magnet of an accelerator. Technical Physics Letters, 43(10), pp. 924-927, 2017.

[12] Preisach, F., On the magnetic aftereffect. IEEE Transactions on Magnetics, 53(3), pp. 1-11, 2017.

[13] Szabó, Z., Tugyi, I., Kádár, G. \& Füzi, J., Identification procedures for scalar Preisach model. Physica B: Condensed Matter, 343(1-4), pp. 142-147, 2004.

[14] Fuzi, J., Analytical approximation of Preisach distribution functions. IEEE Transactions on Magnetics, 39(3), pp. 1357-1360, 2003.

[15] Mayergoyz, I.D., Mathematical Models of Hysteresis and their Applications, Academic Press, 2003.

[16] Vinokurov, N.A. et al., Allowing for hysteresis in the calculation of fields in the elements of accelerator magnetic systems. Technical Physics Letters, 42(7), pp. 708$711,2016$.

[17] Bergqvist, A., Magnetic vector hysteresis model with dry friction-like pinning. Physica B: Condensed Matter, 233(4), pp. 342-347, 1997.

[18] Henrotte, F., Nicolet, A. \& Hameyer, K., An energy-based vector hysteresis model for ferromagnetic materials. COMPEL - The International Journal for Computation and Mathematics in Electrical and Electronic Engineering, 25(1), pp. 71-80, 2006.

[19] Henrotte, F. \& Hameyer, K., A dynamical vector hysteresis model based on an energy approach. IEEE Transactions on Magnetics, 42(4), pp. 899-902, 2006. 